\title{
GRAFICO PER IL CALCOLO DEL PUNTO DI RUGIADA MEDIANTE LO PSICROMETRO ${ }^{*}$;
}

\section{Tenani}

1. - Nella lorma di uso internazionale dei bollettini meteorologrici, l'umidià dell'aria è oggri espressa dal valore del punto di rugiada: e cioe dal valore della temperatura $T_{d}$ a cui l'aria si troverehbe satura qualora venise raffredlata a pressione costante, per semplice sottrazione di calore (ad es. per radiazione), senza modificare il rapporto tra la massa del vapore presente e la massa dellaria secca: la temperatura $T_{1}$ cosi definita $i$ lutlavia quasi identica a quella a cui avviene la condensazione quando in qualsiasi modo l'aria venga raffredlata, perché nella pratica di una tale determinazione le variazioni del suddetto rapporto di mescolanza sono trascurabili.

2. - Conseguentemente la determinazione di $T_{11}$ può eseguirsi in pratica inclifferentemente:

o determinando $T_{\mathrm{d}}$ direltamente con uno psicrometro a condensazione, il che offre le ben note difficolta;

o determinando, collo picrometro, la tensione " del rapore nell'aria e determinando poi, col sussidio di una tavola delle tensioni massime es del vapore, la temperatura $T_{\mathrm{a}}$ per cui $e_{\mathrm{s}}=e$; una tavola di tal genere i costituita precisamente dalla colonna e dell'ordinaria tavola p:icrometrica relativa alla differenza psicrometriea $0^{\circ}, 0$, e dalla colonna contenente le corrispondenti temperature del termometro baEnato (o asciulto);

overo ricorrendo a tavole speciali che forniscano $T_{1}$ in funzione della differenza psicromelrica $J^{t}$ e della temperatura del termometro hagnato (o asciutto).

Lsanclo lo psicrometro, converrà tencr sempre presente che la formula semiempirica che lega il valore di e alla differenza psicrometrica e alla temperatura, varia alquanto con la pressione: a conti fatli si oltiene che, per quote inferiori ai 1000 metri, tenuto conto dei

(*) Comunicazione presentata al Convegno dell Associazione Geofisica Italiana, teruto a Roma il 17-18 giugno 1953. 


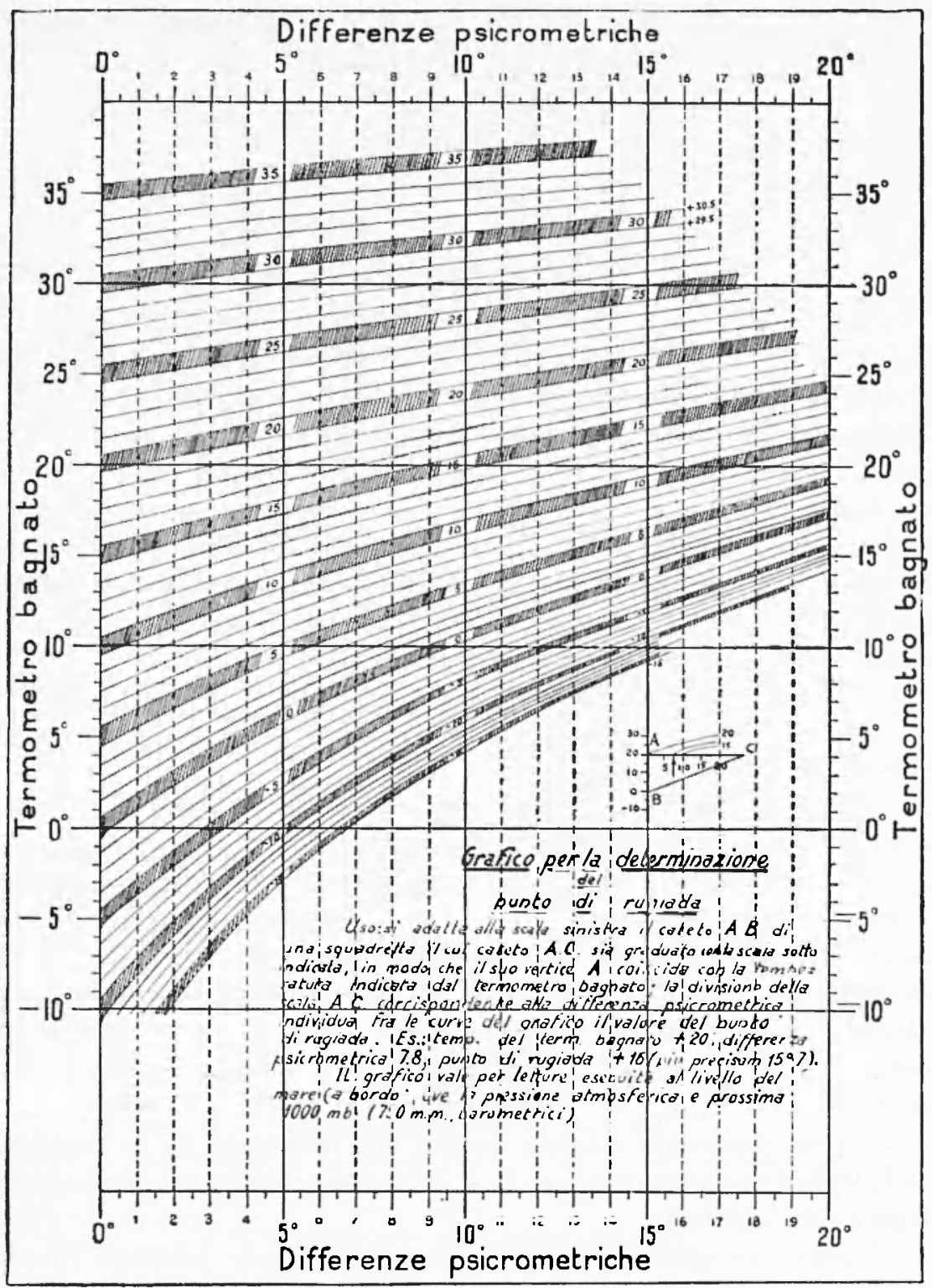

Fig. 1

Grafico per la determinazione del punto di rugiada. 
limiti di precisione delle osservazioni, è possibile trascurare le variazioni della pressione intorno al suo valor medio; e cioè supporre che la pressione ablia il valor medio corrispondente alla quota dello psicrometro.

Analoghe avvertenze dovranno tenersi presenti quando la temperatura è inferiore a $0^{\circ} C$, quando cioc̀ venga a variare la relazione tra $e_{\mathrm{s}}$ e $T_{\mathrm{d}}$.

3. - Un valore approssimato di $T_{\mathrm{d}} \dot{e}$ anche facile a calcolarsi senza uso di tavole, semplicemente:

togliendo dalla temperatura del termometro hagnato la differenza psicrometrica;

ovvero togliendo dalla temperatura del termometro asciutto il doppio della detta differenza;

il risultato risulta tanto più esatto quanto più prossima alla condensazione è l'aria, e cioè quanto più critiche sono le condizioni per la formazione della nebbia.

4. -..- Il nuovo metodo di misurare l'umidita dell'aria ha incontrato particolarmente favore fra i naviganti: soprattutto perché il confronto di $T_{a}$ con la temperatura superficiale dell'acqua offre un metodo comodo e sicuro per giudicare della probabilità di formazione della nebhia. Se la temperatura del mare risulta inferiore a $T_{\mathrm{l}}$, tenendo conto del falto che la rotta si svolga verso regioni in cui il contatto aria-acqua risulti favorevole o contrario a una diminuzione di temperatura dell'aria dal suo valore attuale $T$ al valore $T_{\mathrm{d}}$, il navigante avrà una sicura guida per la previsione della nebbia o per escluderne la formazione: il che avverrà certamente se la temperatura del mare $T_{\mathrm{s}}$ sarà superiore al punto di rugiada $T_{\mathrm{d}}$; se $T_{\mathrm{s}}>T_{\mathrm{d}}$ e $T<T_{\mathrm{d}}$ è prohabile che la nebbia scompaia dopo un breve percorso sul mare.

Nei luoghi in cui la nebhia è frequente un costante confronto di $T_{1}$ con la temperatura superficiale del mare è di estrema utilità.

5. $-T_{\mathrm{tl}}$ rappresenta molte volte pel navigante l'unico modo di eseguire attualmente un apprezzamento della base delle nubi basse: se noi pensiamo che essa si trovi alla quota di condensazione del vapor d'acqua contenuto nell'aria ambiente in seguito a un sollevamento adiahatico, si trova che la base delle condensazioni sarà all'incirca a una quota uquale al prodotlo della differenza (in gradi $C$ ) tra $T$ e $T_{\mathrm{d}}$ per 100-125 metri o, approssimativamente, uguale al prodotto della differenza psicrometrica per 250 metri. 
6. - La regola indicata in 3 per il calcolo del punto di rugiada. esattissima al limite, quando l'aria è satura, perché allora il punto di rugiada è esattamente uquale alla temperatura del termometro bagnato, diventa però rapidamente inesatta al crescere della differenza psicro. metrica. Poiché le tavole destinate a questo computo sono piuttosto voluminose, può essere utile in pratica un piccolo grafico che permette la precisione del decimo di grado, più che sufficiente nella pratica (nei telegrammi meteorici $T_{d}$ è arrotondato al grado, e perciò deve essere per l'appunto conosciuto con la precisione del decimo di gradol. In questo grafico le ordinate sono le temperature del termometro baynato, le ascisse sono le differenze psicrometriche determinate con psicrometri a fionda del genere consigliabile usato sulle nostre navi. Le isoplete tracciate formano tanti viali la cui mezzeria è sul percorso delle isoplete del $T_{\mathrm{a}}$ corrispondenti ai gradi interi. Un piccolo grafico mostra come, con una squadretta si possano eseguire le letture del $T_{t}$ quando siano assegnate la lemperalura del termometro bagnato e la differenza psicrometrica (freccia).

\section{RIASSUNTO}

Si presenta un grafico che permette di determinare il punto di rugiada mediante la differensa psicocrometrica e la temperatura del termometro bagnato.

\section{$S U M M A R Y$}

A graph is presented for determining the dew-point by means of the hygrometric difference and the temperature of the wet thermometer. 